\section{In vivo harmonic generation biopsy of human skin}

\author{
Szu-Yu Chen, Hai-Yin Wu, and Chi-Kuang Sun* \\ National Taiwan University, Graduate Institute of \\ Photonics and Optoelectronics, and Department of Electrical \\ Engineering, Taipei, 10617 Taiwan
}

\begin{abstract}
The ability to in vivo image deep tissues noninvasively with a high resolution is strongly required for optical virtual biopsy. Higher harmonic generation microscopy, combined with second- and third-harmonic generation microscopies, is applied to 17 Asian volunteers' forearm skin. After continuous observation for $30 \mathrm{~min}$, no visible damage was found. Our study proves that harmonic generation biopsy (HGB) is able to satisfy the safety requirement and to provide high penetrability $(\sim 300 \mu \mathrm{m})$ and submicron resolution all at the same time and is a promising tool for future virtual biopsy of skin diseases. In contrast to a previous study on fixed human skin specimens, a much improved penetrability and much reduced resolution-degradation versus depth are found in this in vivo examination. @ 2009 Society of Photo-Optical Instrumentation Engineers. [DOI: 10.1117/1.3269676]
\end{abstract}

Keywords: harmonic generation microscopy; dermatology; noninvasive; in vivo; human skin; high penetrability; high resolution.

Paper 09275LRR received Jul. 1, 2009; revised manuscript received Oct. 9, 2009; accepted for publication Oct. 12, 2009; published online Dec. 28, 2009.

To diagnose skin conditions and diseases, the pathohistological microscopic analysis of excised tissues is most commonly used today. However, the processing-procedureinduced artifacts lead to misdiagnosis, and a painful biopsy process is needed with side effects. A least-invasive tool that can perform high-resolution virtual biopsy is thus desired to assist or replace the physical biopsy for diseases diagnoses, including skin diseases. For early diagnoses of skin cancers and minor skin diseases, virtual skin biopsy is more appropriate. Since the early pathological changes may occur in the dermis layer, an optical virtual biopsy tool that can perform least-invasive imaging deep inside the skin with a submicron resolution is strongly required for pathohistological diagnoses. Recently, optical imaging techniques such as confocal microscopy, ${ }^{1,2}$ two-photon fluorescence (2PF) microscopy, ${ }^{3}$ and second-harmonic generation ( $\mathrm{SHG}$ ) microscopy ${ }^{4}$ have been applied for in vivo skin imaging. However, these techniques are limited by photodamage, lower penetrability, or lower resolution. It is thus highly desired to apply $1230-\mathrm{nm}$ Cr:forsterite $(\mathrm{Cr}: \mathrm{F})$ laser-based higher harmonic generation (HHG) microscopy for least-invasive virtual skin biopsy. Since no electron transition is required in the HHG processes, ${ }^{5}$ light attenuation in human skin reaches a minimum value within 1200 to $1300-\mathrm{nm}$ due to reduced scattering and pigment absorption. ${ }^{6}$ With the same $40-\mathrm{mW}$ excita-

*Address all correspondence to: Chi-Kuang Sun, Tel: 886-2-3366-5085; Fax: 886-2-3366-3614; E-mail: sun@cc.ee.ntu.edu.tw tion power, Cr:F-based SHG microscopy has higher penetrability than Ti:sapphire (Ti:S)-based SHG microscopy. ${ }^{7}$ Through the viability test of mammalian embryo, ${ }^{8}$ a high tolerance of $>140 \mathrm{~mW}(>29 \mathrm{~J})$ can be allowed under Cr:F excitation, while live cells irradiated with 730 to $800-\mathrm{nm}$ beams of $>1 \mathrm{~mW}(0.2 \mathrm{~J})$ were found to inhibit cloning efficiency. ${ }^{9}$ In previous in vivo $\mathrm{Cr}$ :F-based HHG studies, zebrafish embryos with 20 -h continuous observation (100 to $140 \mathrm{~mW}$, $>7 \mathrm{KJ}$ ) can develop normally into the larva stages. ${ }^{10}$ Hamster oral mucosa with 3-h continuous observation in the same area $(150 \mathrm{~mW}, 1620 \mathrm{~J})$ were excised immediately for pathological examination, while no evidence of photodamage appeared. ${ }^{11}$ This much-improved viability versus Ti:S-based (800 nm; $1.55 \mathrm{eV}$ ) systems could be attributed to much-reduced multiphoton absorption with a lower photon energy (1230 nm; $1.01 \mathrm{eV}) .^{12}$

In this paper, real-time in vivo $\mathrm{HHG}$ imaging of Asian skin combined with epi-SHG and epi-third-harmonic generation (epi-THG) modalities is reported. To the best of our knowledge, this is also the first report on the clinical trial of THG imaging. In this study, a Cr:F laser is used, and a syringepump objective is designed to diminish the image blurring due to breathing and heart beating. Even with the image blurring, the submicron spatial resolution was preserved within the whole imaging depth $(>300 \mu \mathrm{m})$ for all volunteers. The safety issue has also been preliminarily confirmed through a standard damage evaluation protocol for clinical trials. By measuring the Cr:F-excited spectra of live human skin (not shown), 2PF was found to be greatly diminished due to the low photon energy of the Cr:F excitation; ${ }^{13}$ while no threephoton fluorescence (3PF) can be observed, since the probability of the fifth-order nonlinear 3PF is several orders lower than that of the third-order nonlinear THG. In contrast to previous HHG imaging of fixed human skin, ${ }^{14,15}$ a higher penetrability with much reduced resolution-degradation versus depth was found. In addition, real-time dynamic information like blood flow can be provided. In this study, HHG imaging is found to satisfy the clinical safety requirement and to provide high penetrability and submicron resolution all at the same time and is an ideal virtual biopsy tool for assisting, minimizing, or even potentially replacing physical biopsy.

Based on the previous epi-HHG microscope, ${ }^{14}$ some modifications were made for the in vivo harmonic generation biopsy (HGB) system. The in vivo HGB of human skin was performed on the ventral forearm skin of 17 volunteers (9 female and 8 male; 21 to 56 years). This clinical trial was approved by IRB of National Taiwan University Hospital. Before, during, and after the HGB, the tested site-ulnar, ventral, upper $1 / 3$ forearm skin-of the volunteers was recorded by photographing. A medical doctor kept checking volunteers' status during HGB. In this trial, the following protocols were applied: (1) the total exposure time was limited to 30 mins in the same area; and (2) two scanning modes were used: a slow mode $(90 \mathrm{~mW} ; 0.37 \mathrm{~Hz})$ and a fast mode $(120 \mathrm{~mW} ; 2 \mathrm{~Hz})$. The accumulated photon energy was around 180 to $200 \mathrm{~J}$ in one area. The tested area was evaluated by a dermatologist immediately, several hours, 24 hours, 3 days, and 1 week after HGB. During HGB, only one volunteer reported a possible stinging sensation for $<1 \mathrm{~s}$, which was claimed to be uncer-

1083-3668/2009/14(6)/060505/3/\$25.00 @ 2009 SPIE 


\section{JBO LETTERS}

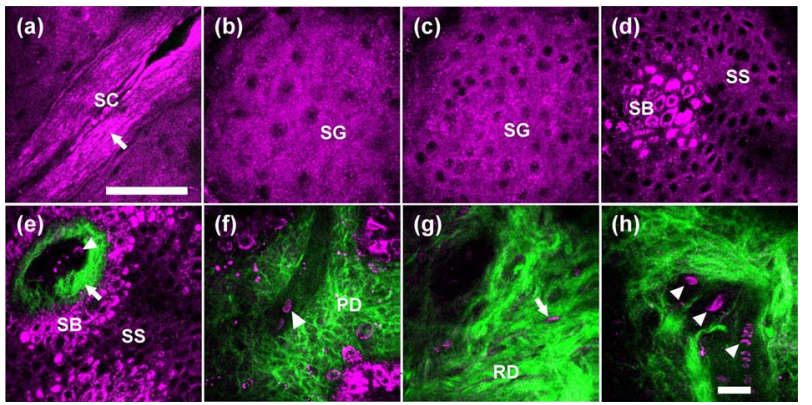

Fig. 1 (a) to (h) In vivo lateral epi-SHG and epi-THG images of human forearm skin (obtained with slow mode) at different depths inward from outside. In epidermis, THG can reveal the (a) multilayer structure of the SC (arrow); (b) and (c) the SG cells at upper and deeper layers; and (d) and (e) the SS and SB cells at the epidermisdermis junction. The collagen fibers in the tip of the papilla can be revealed by SHG microscopy [arrow in (e)]. (f) The loose areolar connective tissue in the PD; (g) and (h) the dense irregular connective tissue in the RD can be shown and distinguished by SHG, while the erythrocytes [arrowheads in (e), (f), and (h)] and fibroblasts [arrow in (g) ] can be observed through THG. THG and SHG are represented by the magenta and green pseudo-colors. Scale bar: $50 \mu \mathrm{m}$. (Color online only.)

tain. There were no inflammatory symptoms, no skin color change, no pigmentation, no wound, no blister formation, and no ulceration reported. The procedure was comfortable according to volunteers' opinions, indicating the feasibility of the HGB for virtual skin biopsy.

Figure 1 shows the lateral SHG and THG images out of a stack of HGB sections from the stratum corneum (SC) to reticular dermis $(\mathrm{RD})$. The depth of the imaging was adjusted by moving the objective in the $z$ direction. Throughout whole imaging depth, the photomultiplier tube (PMT) voltages were sometimes lowered at the stratum basale (SB) due to the excessive THG signals and we can repeatedly achieve $>300 \mu \mathrm{m}$ penetrability in all volunteers. In this in vivo study, THG imaging contrasts were found to be dominated by the interfaces 5 between lipid and corneocytes and the cytoplasmic organelles. ${ }^{8}$ In epidermis, the cellular morphology can be clearly distinguished by THG microscopy, while the THG contrasts can also be found in dermis to show fibroblasts, erythrocytes, collagen fiber bundles, and elastin fibers. ${ }^{16}$ In dermis, collagen-sensitive $\mathrm{SHG}^{5}$ revealed the diverse collag- enous structures in the papillary dermis (PD) and RD. To extend the imaging depth to be much more than $300 \mu \mathrm{m}$, contrast adjustment with higher PMT voltage is needed and was not performed in our protocol. As shown in Fig. 1(a), the multilayer SC structure can be highly resolved through THG, while the nuclei of keratinocytes appeared dark in contrast to the bright cytoplasm due to the efficient THG from cytoplasmic organelles. Figures 1(b) and 1(c) show the squamousshaped stratum granulosum (SG) cells at different depths. At the bumpy epidermis-dermis junction (EDJ), the stratum spinosum (SS) and SB cells were found [Figs. 1(d) and 1(e)] in the same section, and the collagen fibers in the dermal papilla were revealed by SHG. In Figs. 1(b)-1(d), the average nuclear diameter (ND) of the keratinocytes at different depths can be analyzed to be $6.8,5.5$, and $4.9 \mu \mathrm{m}$, while the average internuclear distance (IND) was analyzed to be 19.9, 10.2, and $8.4 \mu \mathrm{m}$, respectively. Analyzing the IND versus depth, an exponential decreasing of the IND with increasing depth can be observed [Fig. 2(a)], and this progressive change of the IND agrees with the histological results, ${ }^{17}$ indicating the normal keratinization process. This in vivo cytological information has its diagnostic significance for skin diseases with loss of normal keratinization. In dermis, Fig. 1(f) shows the loose areolar collagen fibers in the PD, while Figs. 1(g) and 1(h) show the dense reticular collagen fibers in the RD. Due to the THG contrast from oxyhemoglobin ${ }^{18}$ and cytoplasmic organelles, the erythrocytes and the fibroblasts can all be revealed by THG. ${ }^{19}$ By fixing the imaging depth for a real-time continuous monitoring (Video 1), the erythrocytes can be easily identified due to their movement, size, and shapes. The contrast of the fibroblast was confirmed by a comparison between HGB images and histological images of surgical specimens. Compared with confocal microscopy, ${ }^{20}$ the erythrocytes and the fibroblast can be distinguished from the collagen fibers more easily under HGB due to the simultaneous SHG contrast. Right at the surface of the skin, the lateral resolution of THG microscopy can be analyzed from the multilayer structure of the SC [arrow in Fig. 2(b)] to be $0.45 \pm 0.05 \mu \mathrm{m}$, while in the dermis, the lateral resolution of SHG microscopy can be analyzed from collagen fibrils [arrow in Fig. 2(c); at $200 \mu \mathrm{m}$ deep] to be $0.92 \pm 0.12 \mu \mathrm{m}$, with corresponding THG resolution of $0.75 \pm 0.1 \mu \mathrm{m}$. Figure 2(d) shows this analyzed lateral resolution of THG microscopy versus depth, averaged with data of six volunteers (20 to 30 years). Com-
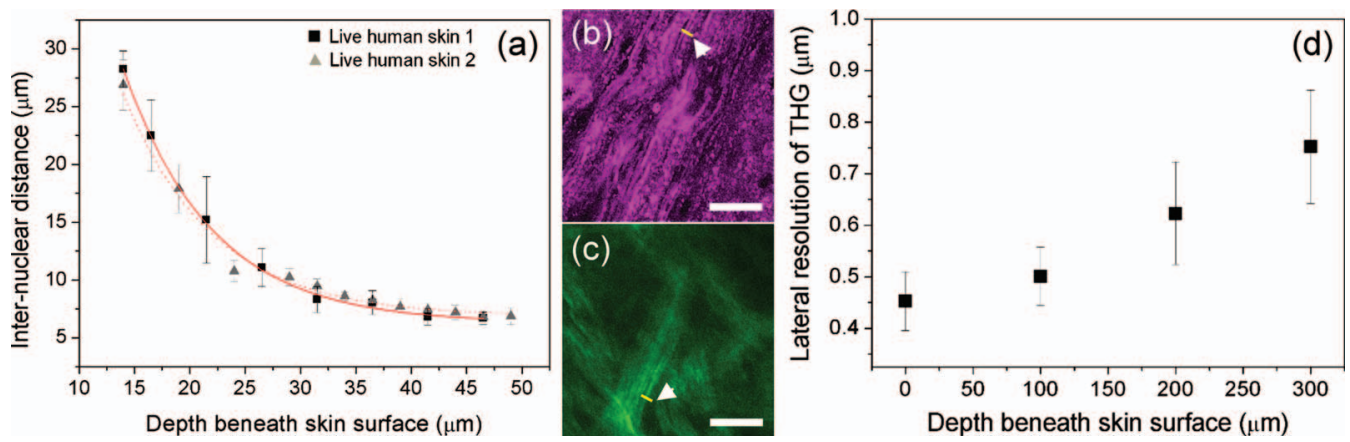

Fig. 2 (a) Internuclear distances (INDs) versus depth analyzed from in vivo epi-THG images. The lateral resolution of THG microscopy right at skin surface can be analyzed from (b) multilayer stratum corneum (arrow), while the lateral resolution of SHG microscopy can be analyzed from (c) collagen fibrils (arrow; $200 \mu \mathrm{m}$ deep). (d) The lateral resolution of THG microscopy versus depth. THG and SHG are represented by the magenta and green pseudo-colors. Scale bar: $50 \mu \mathrm{m}$. 


\section{JBO LETTERS}

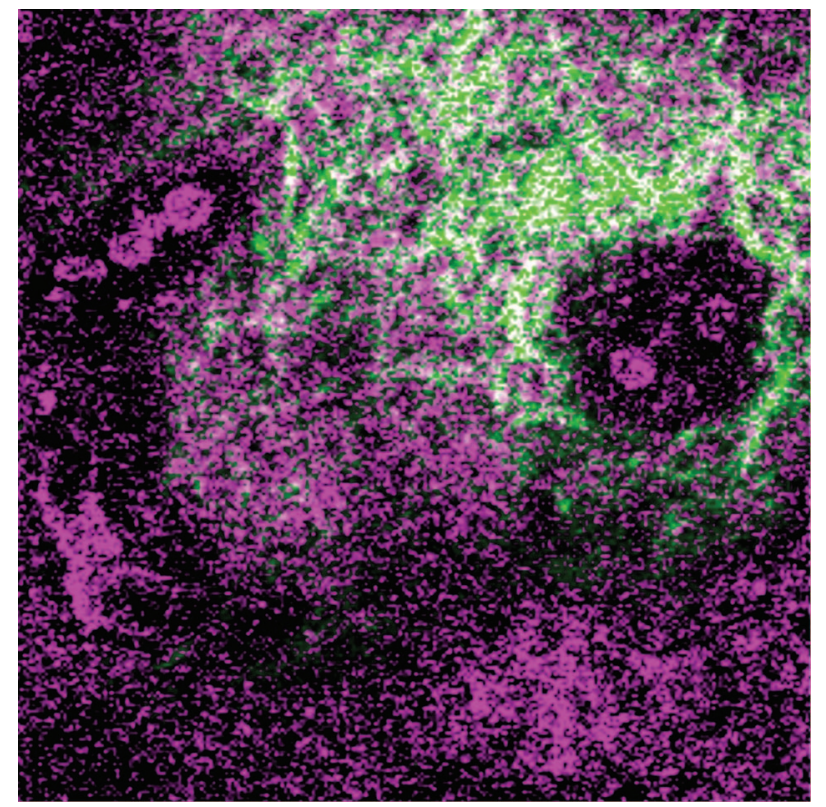

Video 1 In vivo movie of erythrocytes flowing in dermal capillaries. The movie is recorded by continuous real-time imaging with a fast scanning mode (frame rate: $2 \mathrm{~Hz}$ ) at a fixed depth. Image size: $80 \times 80 \mu \mathrm{m}^{2}$ (QuickTime, 4.3 MB).

[URL: http://dx.doi.org/10.1117/1.3269676.1]

pared with the previous analysis in fixed human skin, ${ }^{15}$ our in vivo study shows a much reduced resolution degradation versus depth, indicating much reduced point-spread function aberration $^{21}$ of the $1230-\mathrm{nm}$ excitation light in the live tissues versus fixed tissues.

In conclusion, in vivo real-time HGB of human skin has been demonstrated. Even with the image blurring resulting from vibrations, a submicron lateral resolution of THG microscopy was preserved at a depth of $\sim 300 \mu \mathrm{m}$, achieved without increasing the PMT voltage. In contrast to the previous fixed skin imaging, the in vivo HGB of human skin showed much reduced resolution-degradation versus depth while providing real-time dynamic information in the live tissues. Through the damage evaluation, the noninvasiveness of this imaging tool has been preliminarily proved. Combined with the high spatial resolution, high penetrability, and various imaging capabilities, IR-based HHG imaging will be a promising tool for future noninvasive virtual biopsy of skin diseases.

\section{Acknowledgments}

We acknowledge assistance from Dr. Yi-Hua Liao and Dr. Wen-Jeng Lee of NTUH. This research is sponsored by the National Health Research Institute of Taiwan (NHRI-EX989201EI), NTU Research Center for Medical Excellence, and NTU Grant No. 98R0036-01.

\section{References}

1. B. R. Masters and P. T. C. So, "Confocal microscopy and multiphoton excitation microscopy of human skin in vivo," Opt. Express 8 , 2-10 (2001).
2. K. S. Nehal, D. Gareau, and M. Rajadhyaksha, "Skin imaging with reflectance confocal microscopy," Semin Cutan Med. Surg. 27, 37-43 (2008).

3. B. R. Masters, P. T. C. So, and E. Gratton, "Multiphoton excitation fluorescence microscopy and spectroscopy of in vivo human skin," Biophys. J. 72, 2405-2412 (1997).

4. M. J. Koehler, K. Konig, P. Elsner, R. Buckle, and M. Kaatz, "In vivo assessment of human skin aging by multiphoton laser scanning tomography," Opt. Lett. 31, 2879-2881 (2006).

5. C.-K. Sun, "Higher harmonic generation microscopy," Adv. Biochem. Eng./Biotechnol. 95, 17-56 (2005).

6. R. R. Anderson and J. A. Parish, "The optics of human skin," J. Invest. Dermatol. 77, 13-19 (1981).

7. T. Yasui, Y. Takahashi, M. Ito, S. Fukushima, and T. Araki, "Ex vivo and in vivo second-harmonic-generation imaging of dermal collagen fiber in skin: comparison of imaging characteristics between modelocked Cr:forsterite and Ti:sapphire lasers," Appl. Opt. 48, D88-D95 (2009).

8. C.-S. Hsieh, S.-U. Chen, Y.-W. Lee, Y.-S. Yang, and C.-K. Sun, "Higher harmonic generation microscopy of in vitro cultured mammal oocytes and embryos," Opt. Express 16, 11574-11588 (2008).

9. K. Konig, P. T. C. So, W. W. Mantulin, and E. Gratton, "Cellular response to near-infrared femtosecond laser pulses in two-photon microscopes," Opt. Lett. 22, 135-136 (1997).

10. S.-Y. Chen, C.-S. Hsieh, and S.-W. Chu, "Noninvasive harmonics optical microscopy for long-term observation of embryonic nervous system development in vivo," J. Biomed. Opt. 11, 054022 (2006).

11. S.-P. Tai, W.-J. Lee, D.-B. Shieh, P.-C. Wu, H.-Y. Huang, C.-H. Yu, and C.-K. Sun, "In vivo optical biopsy of hamster oral cavity with epi-third-harmonic-generation microscopy," Opt. Express 14, 61786187 (2006).

12. I.-H. Chen, S.-W. Chu, C.-K. Sun, P.-C. Cheng, and B.-L. Lin, "Wavelength dependent damage in biological multiphoton confocal microscopy: a micro-spectroscopic comparison between femtosecond Ti:sapphire and Cr:forsterite laser sources," Opt. Quantum Electron. 34, 1251-1266 (2002)

13. J.-H. Lee, S.-Y. Chen, S.-W. Chu, L.-F. Wang, C.-K. Sun, and B.-L. Chiang, "Noninvasive in vivo assessment of epidermal hyperkeratosis and dermal fibrosis in atopic dermatitis," J. Biomed. Opt. 14, 014008 (2009).

14. S.-P. Tai, T.-H. Tsai, W.-J. Lee, D.-B. Shieh, Y.-H. Liao, H.-Y. Huang, K. Zhang, H.-L. Liu, and C.-K. Sun, "Optical biopsy of fixed human skin with backward collected optical harmonics signals," Opt. Express 13, 8231-8242 (2005).

15. T.-H. Tsai, S.-P. Tai, W.-J. Lee, H.-Y. Huang, Y.-H. Liao, and C.-K. Sun, "Optical signal degradation study in fixed human skin using confocal microscopy and higher-harmonic optical microscopy," Opt. Express 14, 749-758 (2006).

16. C.-H. Yu, S.-P. Tai, C.-T. Kung, I.-J. Wang, H.-C. Yu, H.-J. Huang, W.-J. Lee, Y.-F. Chan, and C.-K. Sun, "In vivo and ex vivo imaging of intra-tissue elastic fibers using third-harmonic-generation microscopy," Opt. Express 15, 11167-11177 (2007).

17. R. R. Wickett and M. O. Visscher, "Structure and function of the epidermal barrier," Am. J. Infect. Control 34, S98-S110 (2006).

18. S.-Y. Chen, S.-P. Tai, C.-S. Hsieh, C.-Y. Chen, C.-H. Yu, Y.-W. Lee, W.-J. Lee, S.-U. Chen, F.-H. Chang, and C.-K. Sun, "Least invasive in vivo imaging using harmonic generation microscopy," Proc. SPIE 6860, 686002-11 (2008).

19. J. A. Palero, H. S. de Bruijn, A. V. van den Heuvel, H. J. C. M Sterenborg, and H. C. Gerritsen, "Spectrally resolved multiphoton imaging of in vivo and excised mouse skin tissues," Biophys. J. 93 , 992-1007 (2007)

20. J. Seylaz, R. Charbonne, K. Nanri, D. Von Euw, J. Borredon, K. Kacem, P. Meric, and E. Pinard, "Dynamic in vivo measurement of erythrocyte velocity and flow in capillaries and of microvessel diameter in the rat brain by confocal laser microscopy," J. Cereb. Blood Flow Metab. 19, 863-870 (1999).

21. M. Gu, X. Gan, A. Kisteman, and M.-G. Xu, "Comparison of penetration depth between two-photon excitation and single-photon excitation in imaging through turbid tissue media," Appl. Phys. Lett. 77, 1551-1553 (2000). 